\title{
THE MEASURABILITY OF A STOCHASTIC PROCESS OF SECOND ORDER AND ITS LINEAR SPACE
}

\author{
STAMATIS CAMBANIS 1
}

\begin{abstract}
It is of considerable theoretical and practical interest to know whether a stochastic process has a measurable modification. For the important class of second order processes, simple necessary and sufficient conditions for the existence of a measurable modification are given in terms of the autocorrelation of the process and the separability of its reproducing kernel Hilbert space or its linear space. It is shown that weakly continuous processes, processes with orthogonal increments and second order martingales always have measurable modifications. Also necessary and sufficient conditions are given in terms of integral representations for the linear space of a second order process to be separable. As a consequence it is shown that a second order process is oscillatory if and only if its linear space is separable.
\end{abstract}

1. The measurability of a stochastic process of second order. Let $T$ be a complete separable metric space or a Borel subset of a complete separable metric space, and $\mathcal{B}(T)$ the $\sigma$-algebra of Borel sets of $T$, and let $X, t \in T$, be a real stochastic process on the probability space $(\Omega, \mathcal{F}, P)$, where real means $[-\infty,+\infty]$-valued. $X_{t}, t \in T$, is called measurable if the map $(t, \omega) \rightarrow X_{t}(\omega)$ is $(\mathscr{B}(T) \times \mathfrak{F})$-measurable. A process $Y_{t}, t \in T$, on $(\Omega, \mathfrak{F}, P)$ is called a mudification of $X_{t}, t \in T$, if $P\left\{X_{t}=Y_{t}\right\}=1$ for all $t$ in $T$. $X_{t}, t \in T$, is of second order if $E\left(X_{t}^{2}\right)<+\infty$ for all $t$ in $T$, and then its autocorrelation $R$ is defined by $R(t, s)=E\left(X_{t} X_{s}\right)$ for all $t, s$ in $T$. It is clear from Fubini's theorem that if a second order process $X_{t}, t \in T$, has a measurable modification, then $R$ is $(\mathcal{B}(T) \times \mathfrak{B}(T))$-measurable. That the measurability of $R$ is not sufficient for the existence of a measurable modification of $X_{t}, t \in T$, is demonstrated in Remark 1. It is thus of interest to find a condition which along

Received by the editors September 4, 1973 and, in revised form, February 11, 1974.

AMS (MOS) subject classifications (1970). Primary 60G05, 60G99.

Key words and phrases. Second order processes, measurable processes, weakly continuous processes, processes with orthogonal increments, second order martingales, the linear space of a process, integral representations, oscillatory processes.

1 This research was supported by the Air Force Office of Scientific Research under Grant AF OSR-68-1415. 
with the measurability of $R$ would imply the existence of a measurable modification of $X_{t}, t \in T$. This question is answered in Theorem 1 , where in fact necessary and sufficient conditions are given for a second order process to have a measurable modification. As a consequence of these conditions, the existence of a measurable modification of a second order process is a second order property; a fact that is also true for general (not necessarily second order) processes [10].

The proof of Theorem 1 is based on the necessary and sufficient condition for a process (not necessarily of second order) to have a measurable modification given in [2], which is expressed as follows (here the terminology of [3] is followed). Let $M$ be the space of all real random variables on $(\Omega, \mathcal{F}, P)$ with the topology of convergence in probability, where random variables that are equal a.e. $[P]$ are considered identical. If $\xi$ is a real random variable, its class in $M$ is denoted by [ $\xi$ ]. Then $X_{t}, t \in T$, has a measurable modification if and only if the map from $T$ to $M$ taking $t$ to $\left[X_{t}\right]$ is measurable [2], [3] (in which case it has separable range). Moreover, the measurable modification can be taken to be separable and also progressively measurable, the latter if $T$ is an interval and a nondecreasing family $\mathcal{F}_{t}, t \in T$, of sub- $\sigma$-algebras of $\mathcal{F}$ is given.

For a second order process $X_{t}, t \in T$, we denote by $H(X)$ the closure in $L_{2}(\Omega, \mathcal{F}, P)$ of the linear space of the random variables $\left\{X_{t}, t \in T\right\}$, and we call it the linear space of the process. We also denote by $\mathscr{R}(R)$ the reproducing kernel Hilbert space of the real, symmetric, nonnegative definite function $R$. It is well known that $R(R)$ consists of all functions $f$ on $T$ of the form $f(t)=E\left(\xi X_{t}\right), t \in T$, for some $\xi \in H(X)$, and that the map $\xi \rightarrow E\left(\xi X_{t}\right)$ defines an inner product preserving isomorphism between $H(X)$ and $R(R)$ [13, p. 302].

Theorem 1. Let $X_{t}, t \in T$, be a real, second order process with autocorrelation $R$. The following are equivalent.

(i) $X_{t}, t \in T$ has a measurable modification.

(ii) $R$ is $(\mathcal{B}(T) \times \mathcal{B}(T)$ )-measurable, and $H(X)($ or $\mathcal{R}(R))$ is separable.

Proof. We first show that (ii) implies (i). It suffices to show that the map $X: T \rightarrow M$ defined by $X(t)=\left[X_{t}\right]$ is measurable; the construction of a modification is the same as in [2] or in [3]. The metric $\rho$ on $M$, defined by

$$
\rho(\xi, \eta)=E(|\xi-\eta| /(1+|\xi-\eta|))
$$


metrizes the topology of convergence in probability. Thus for the measurability of $X$, it suffices to show that (a) $X^{-1}(B) \in \mathcal{F}$ for every set $B$ in $M$ of the form $B=\{\eta \in M: \rho(\eta, \xi) \leq r\}$, where $\xi \in M$ and $r>0$, and (b) for every compact subset $K$ of $T$ there is a countable subset $C$ of $M$ such that $\left[X_{t}\right] \epsilon$ $\bar{C}$, the closure of $C$ for every $t \in K$ [1, Chapter IV, p. 191, Chapter IX, p. 14]. (b) follows from the separability of $H(X)$ and the fact that convergence in $L_{2}(\Omega, \mathcal{F}, P)$ implies convergence in probability; any countable dense subset $C$ of $H(X)$ satisfies (b) for all compact subsets of $T$. Since $X^{-1}(B)=$ $\left\{t \in T: \rho\left(\left[X_{t}\right], \xi\right) \leq r\right\}$, for (a) it suffices to prove that the real function $\rho\left(\left[X_{t}\right], \xi\right)$ on $T$ is $\mathfrak{B}(T)$-measurable for all $\xi \in M$.

Let $\left\{\xi_{n}\right\}_{n=1}^{\infty}$ be a complete orthonormal sequence in $H(X)$ (which exists because $H(X)$ is separable). Then for all $t \in T$ we have

$$
X_{t}=\sum_{n=1}^{\infty} a_{n}(t) \xi_{n}
$$

in $L_{2}(\Omega, \mathcal{F}, P)$, where $a_{n}(t)=E\left(\xi_{n} X_{t}\right)$. Thus $a_{n} \in R(R)$. If for every $t \in T$ we let $X_{t}^{(N)}=\sum_{n=1}^{N} a_{n}(t) \xi_{n}$, then $X_{t}^{(N)}$ converges to $X_{t}$ in $L_{2}(\Omega, \mathcal{F}, P)$, and thus in probability. Hence

$$
\rho\left(\left[X_{t}\right], \xi\right)=\lim _{N \rightarrow \infty} \rho\left(\left[X_{t}^{(N)}\right], \xi\right)
$$

for all $t \in T$. Note that every function in $R(R)$ is either a finite linear combination of the functions $\{R(t, \cdot), t \in T\}$ or a pointwise limit on $T$ of such functions. Hence, since $R$ is $(\mathfrak{B}(T) \times \mathfrak{B}(T))$-measurable, $R(t, \cdot)$ is $\mathscr{B}(T)$ measurable for all $t \in T$, and it follows that every function in $R(R)$ is $\mathscr{B}(T)$ measurable. Consequently $X_{t}^{(N)}(\omega)$ is $(\mathfrak{B}(T) \times \mathcal{F})$-measurable. By Fubini's theorem,

$$
\rho\left(\left[X_{t}^{(N)}\right], \xi\right)=E\left(\left|X_{t}^{(N)}-\xi\right| /\left(1+\left|X_{t}^{(N)}-\xi\right|\right)\right)
$$

is $\mathcal{B}(T)$-measurable, and thus so is $\rho\left(\left[X_{t}\right], \xi\right)$, which completes the proof.

We now show that (i) implies (ii). The measurability of $R$ follows from Fubini's theorem and (i). In order to prove the separability of $H(X)$ we first assume that $R$ is uniformly bounded on $T$. Now (i) implies that $\left\{\left[X_{t}\right], t \in T\right\}$ is separable in $M[3]$. Thus there exists a countable subset $N$ of $\left\{\left[X_{t}\right]\right.$, $t \in T\}$ such that for every fixed $t$ in $T,\left[X_{t}\right]$ is the limit in probability of a sequence $\left\{\xi_{n}\right\}_{n=1}^{\infty}$ in $N$. Since the uniform boundedness of $R$ on $T$ implies that $N$ is a bounded set in $L_{2}(\Omega, \mathcal{F}, P)$, it follows that $\left\{\xi_{n}\right\}_{n=1}^{\infty}$ converges 
to $\left[X_{t}\right]$ weakly in $L_{2}(\Omega, \mathcal{F}, P)\left[9\right.$, p. 207], and thus $\left[X_{t}\right]$ belongs to the closure in $L_{2}(\Omega, \mathcal{F}, P)$ of the linear manifold generated by $\left\{\xi_{n}\right\}_{n=1}^{\infty}[8, \mathrm{p} .68]$. It follows that $H(X)$ is equal to the closure in $L_{2}(\Omega, \mathcal{F}, P)$ of the linear manifold generated by $N$ and, since $N$ is countable, $H(X)$ is separable.

We now consider the general case, and define for $N=1,2, \cdots, T_{N}=$ $\{t \in T: R(t, t) \leq N\}$. Since $R$ is measurable, $T_{N} \in \mathcal{B}(T)$, and by (i) $\left\{X_{t}\right.$, $\left.t \in T_{N}\right\}$ has a measurable modification. It follows, by what has been proven, that the $L_{2}(\Omega, \mathcal{F}, P)$ closure of the linear span of the random variables $\left\{X_{t}, t \in T_{N}\right\}, H_{N}(X)$, is separable. Since $X_{t}$ is of second order, $R$ is finite valued and thus $T_{N} \uparrow T$. It follows that $H(X)$ is the $L_{2}(\Omega, \mathcal{F}, P)$ closure of $\bigcup_{N=1}^{\infty} H_{N}(X)$, and thus $H(X)$ is separable.

Thus a $(\mathscr{B}(T) \times \mathfrak{B}(T))$-measurable, symmetric, nonnegative definite, real function $R$ on $T \times T$ is the autocorrelation of a measurable process if and only if $R(R)$ is separable. For Gaussian processes it can be easily shown that (ii) implies (i) without relying on the results of [3]; this is done in [12, p. 44].

It should be pointed out that a weakly stationary process on the real line has a measurable modification if and only if its autocorrelation function is continuous. The "if" part is well known and a simple proof of the "only if" part is given in [6, pp. 559-560].

Remark 1. Let $T=[0,1]$ and $R(t, s)=1$ for $t=s$ in $T$, and $R(t, s)$ $=0$ for $t \neq s$ in $T$. Since $R$ is symmetric and nonnegative definite, there exists a probability space $(\Omega, \mathcal{F}, P)$ and a real process $X_{t}, t \in T$, on it with autocorrelation $R, R$ is clearly $(\mathcal{B}(T) \times B(T)$ )-measurable, but since the values of $X_{t}$ are orthogonal in $L_{2}(\Omega, \mathcal{F}, P), E\left(X_{t} X_{s}\right)=0$ for $t \neq s$ in $T, H(X)$ is not separable, and by Theorem $1, X_{t}, t \in T$, does not have a measurable modification. This can be also shown without using Theorem 1. Indeed assume that $X_{t}, t \in T$, has a measurable modification $Y_{t^{\prime}} t \in T$. Then

$$
E\left(\int_{0}^{1} Y_{t}^{2} d t\right)=\int_{0}^{1} R(t, t) d t=1
$$

implies that $\int_{0}^{1} Y_{t}^{2} d t<+\infty$ a.e. $[P]$. If $\left\{\phi_{n}\right\}_{n=1}^{\infty}$ is a complete orthonormal set in $L_{2}(T)=L_{2}(T, \mathcal{B}(T), L e b)$, then

$$
Y_{t}=\sum_{n=1}^{\infty} \xi_{n} \phi_{n}(t)
$$


in $L_{2}(T)$ a.e. $[P]$, where $\xi_{n}=\int_{0}^{1} Y_{t} \phi_{n}(t) d t$ a.e. $[P]$. Then

$$
E\left(\xi_{n}^{2}\right)=\int_{0}^{1} \int_{0}^{1} R(t, s) \phi_{n}(t) \phi_{n}(s) d t d s=0
$$

i.e., $\xi_{n}=0$ a.e. $[P]$, and thus $\int_{0}^{1} Y_{t}^{2} d t=\Sigma_{n=1}^{\infty} \xi_{n}^{2}=0$ a.e. [P] which contradicts $E\left(\int_{0}^{1} Y_{t}^{2} d t\right)=1$. It follows that $X_{t}, t \in T$, does not have a measurable modification.

Corollary 1. A second order process $X_{t}, t \in T$, which satisfies any of the following conditions has a measurable modification (in (iii) also progres. sively measurable).

(i) $X_{t}, t \in T$, is weakly continuous on $T$.

(ii) $T$ is an arbitrary interval and $X_{t}, t \in T$, has orthogonal increments.

(iii) $T$ is an arbitrary interval and $X_{t}, t \in T$, is a martingale.

Proof. (i) Since $T$ is separable and $X_{t}$ weakly continuous on $T, H(X)$ is separable [13, p. 272]. By the weak continuity of $X_{t}, R(t, \cdot)$ is continuous, hence $B(T)$-measurable, for all $t$ in $T$. The conclusion follows as in the first part of the proof of Theorem 1.

(ii) It is known that $H(X)$ is separable [5, p. 110]. Also, that $X_{t}$ has left and right $L_{2}(\Omega, \mathcal{F}, P)$ limits on $T$ and that, except on a countable subset of $T, X_{t^{-}}=X_{t}=X_{t^{+}}$. This implies the measurability of $R$ and the result follows from Theorem 1 .

(iii) Define the function $F$ by $F(t)=E\left(X_{t}^{2}\right)$ for all $t$ in $T$. By the martingale property, with respect to the nondecreasing family $\mathcal{F}_{t}, t \in T$, of sub- $\sigma$-algebras of $\mathcal{F}$, we have, for all $s \leq t$ in $T$,

$$
E\left(X_{t} X_{s}\right)=E\left[E\left(X_{t} X_{s} / F_{s}\right)\right]=E\left[X_{s} E\left(X_{t} / F_{s}\right)\right]=E\left(X_{s}^{2}\right)
$$

and thus

$$
E\left(\left\{X_{t}-X_{s}\right\}^{2}\right)=F(t)-F(s)
$$

It follows from this relationship, as in $[5, \mathrm{p} .110]$ and in (ii), that $H(X)$ is separable and $R$ is $(\mathscr{B}(T) \times \mathscr{B}(T))$-measurable.

We conclude this section with a property which is useful in connection with problems involving conditional probabilities; such as, for instance, the existence of jointly measurable densities (see [7, pp. 616-617]) and properties related to metric transitivity (see [15, Chapter IV.8]). A $\sigma$-algebra is called separable if it is generated by a countable class of sets. A sub- $\sigma$ algebra $\mathfrak{F}^{\prime}$ of $\mathcal{F}$ is said to coincide $\bmod 0$ with the $\sigma$-algebra $\mathcal{F}$ if for 
every set $E$ in $\mathcal{F}$ there is a set $E^{\prime}$ in $\mathcal{F}^{\prime}$ such that $P\left(E \Delta E^{\prime}\right)=0$. Let $\mathcal{F}(X)$ be the sub- $\sigma$-algebra of $\mathcal{F}$ generated by the random variables $\left\{X_{t}\right.$, $t \in T\}$. It is known that if $X_{t}$ is continuous in probability on $T, \mathcal{F}(X)$ coincides mod 0 with a separable $\sigma$-algebra. Corollary 2 generalizes this result (and in fact, as it is clear from [3], it is valid for any process with values in a compact metric space).

Corollary 2. If a real process $X_{t}, t \in T$, has a measurable modifica. tion, then $\mathfrak{F}(X)$ coincides mod 0 with a separable $\sigma$-algebra.

Proof. Since $X_{t}, t \in T$, has a measurable modification, $\left\{\left[X_{t}\right], t \in T\right\}$ is a separable subset of $M[3]$. Thus there exists a countable subset $M^{\prime}=$ $\left\{\left[X_{t}\right], t \in S\right\}$ of $\left\{\left[X_{t}\right], t \in T\right\}(S$ is a countable subset of $T)$ such that for every $t$ in $T,\left[X_{t}\right]$ is the limit in probability of a sequence from $M^{\prime}$, and thus $X_{t}$ is the a.e. [P] limit of a sequence from $\left\{X_{t}, t \in S\right\}$. If $\mathcal{F}^{\prime}$ is the sub- $\sigma$-algebra of $\mathcal{F}$ generated by the random variables $\{X, t \in S\}$, then $\mathcal{F}^{\prime} \subseteq \mathcal{F}(X), \mathcal{F}^{\prime}$ is separable and $\mathcal{F}(X)$ coincides with $\mathcal{F}^{\prime} \bmod 0$.

2. On the separability of the linear space of a second order process. The linear space $H(X)$ of a second order process $X, t \in T$, plays an important role in the structure of such processes and in a variety of problems in statistical inference. If $H(X)$ is separable, then $X_{t}$ admits series representations and also integral representations (Theorem 2) that can be effectively used in problems such as linear mean square estimation. Also, the separability of $H(X)$ is the only condition needed for a second order process to have the Hida-Cramer representation. It is thus of interest that a measurable second order process has a separable linear space. $H(X)$ is known to be separable when the process $X, t \in T$, is weakly continuous $[13, \mathrm{p} .272]$, has orthogonal increments $[5, \mathrm{p} .110]$, or is a martingale (Corollary 1(iii)). In Theorem 2, necessary and sufficient conditions are given for $H(X)$ to be separable in terms of integral representations of $X_{t}$

Before starting the theorem, we mention a few basic facts about random measures that can be found for instance in [4], [13]. A random measure $Z$ on a measurable space $(U, \eta)$ is a countably additive map from $U$ to $L_{2}(\Omega, \mathcal{F}, P)$; i.e., whenever $A$ is the disjoint union of the sets $A_{n} \in \mathcal{U}$, $Z(A)=\Sigma_{n=1}^{\infty} Z\left(A_{n}\right)$ in $L_{2}(\Omega, \mathcal{F}, P)$. (Here we consider the case where $Z$ is defined on the entire $\sigma$-algebra $\mathcal{U}$.) To each random measure $Z$ on $U$ with finite variation, there corresponds a finite signed measure $\mu$ on 
$\mathrm{U} \times \mathrm{U}$ by $\mu(A \times B)=E[Z(A) Z(B)], A, B \in \mathcal{U} . \mu$ is symmetric and nonnegative definite on the measurable rectangles of $U \times U$. A random measure $Z$ is called orthogonal if $\mu(A \times B)=0$ whenever $A$ and $B$ are disjoint, and to each orthogonal random measure there corresponds a finite nonnegative measure $\nu$ on $\mathcal{U}$ by $\nu(A)=E\left[Z^{2}(A)\right], A \in \mathcal{U}$. Let $H(Z)$ be the closure in $L_{2}(\Omega, \mathcal{F}, P)$ of the linear span of $\{Z(A), A \in \mathcal{U}\}$, and let $\Lambda_{2}(\mu)$ be the Hilbert space of real, U-measurable functions on $U$ with inner product

$$
\langle f, g\rangle_{\mathbf{\Lambda}_{2}(\mu)}=\int_{U} \int_{U} f(u) g(v) d \mu(u, v)
$$

(of course $\Lambda_{2}(\mu)$ consists of equivalence classes of functions, two functions $f$ and $g$ considered identical if $\left.\langle f-g, f-g\rangle_{\Lambda_{2}(\mu)}=0\right)$. There is an inner product preserving isomorphism between $\Lambda_{2}(\mu)$ and $H(Z)$, denoted by $\leftrightarrow$, such that $I_{A} \leftrightarrow Z(A), A \in \mathcal{U}$, and integration of functions in $\Lambda_{2}(\mu)$ with respect to $Z$ is defined by $\xi=\int_{U} f(u) d Z(u)$, where $f \leftrightarrow \xi$. If $Z$ is orthogonal, there is an inner product preserving isomorphism between $L_{2}(\nu)=L_{2}(U, \mathrm{U}, \nu)$ and $H(Z)$, denoted again by $\leftrightarrow$, such that $I_{A} \leftrightarrow Z(A)$, $A \in \mathrm{U}$, and integration of functions in $L_{2}(\nu)$ with respect to $Z$ is defined by $\xi=\int_{U} f(u) d Z(u)$, where $f \leftrightarrow \xi$.

Theorem 2. Let $X_{t}, t \in T$, be a second order process.

(i) If $H(X)$ is separable, then for every finite measure space $(U, U, \nu)$ such that $L_{2}(\nu)=L_{2}(U, U, \nu)$ is separable and infinite dimensional, $X_{t}$ has a representation

$$
X_{t}=\int_{U} f(t, u) d Z(u) \text { for all } t \text { in } T,
$$

where $Z$ is an orthogonal measure on II with corresponding measure $\nu$ and $f(t, \cdot) \in L_{2}(\nu)$ for all $t$ in $T$. Conversely, if $X_{t}$ has such a representation, $H(X)$ is separable.

(ii) If $H(X)$ is separable, then for every measurable space $(U, U)$ and every finite signed measure $\mu$ on $\mathrm{U} \times \mathrm{U}$ which is symmetric and nonnegative definite on the measurable rectangles of $\mathrm{U} \times \mathrm{U}$, and such that $\Lambda_{2}(\mu)$ is separable and infinite dimensional, $X_{t}$ has a representation (1) where $Z$ is a random measure on II with corresponding measure $\mu$ and $f(t, \cdot) \in \Lambda_{2}(\mu)$ for all $t$ in $T$. Conversely, if $X_{t}$ has such a representation with $Z$ of finite variation, $H(X)$ is separable.

Proof. (i) being a particular case of (ii), we will prove only (ii). If $X_{t}$ has such a representation then $X_{t} \in H(Z)$ for all $t$ in $T$, hence 
$H(X) \subseteq H(Z)$ and the conclusion follows from the isomorphism between $H(Z)$ and $\Lambda_{2}(\mu)$ and the separability of the latter. We now prove the first claim. Assume that $H(X)$ is separable and let $\left\{\xi_{n}\right\}_{n=1}^{\infty}$ and $\left\{f_{n}\right\}_{n=1}^{\infty}$ be complete orthonormal sets in $H(X)$ and $\Lambda_{2}(\mu)$, respectively. Let $K$ be the inner product preserving isomorphism between $\Lambda_{2}(\mu)$ and $H(X)$ such that $K f_{n}=\xi_{n}$. For every $A \in \mathcal{U}$ define $\dot{Z}(A)=K I_{A}$, where $I_{A}$ is the characteristic function of the set $A$. Then clearly $Z$ is countably additive and for all $A, B \in \mathcal{U}$,

$$
E[Z(A) Z(B)]=\left\langle I_{A}, I_{B}\right\rangle_{\Lambda_{2}(\mu)}=\mu(A \times B),
$$

i.e., $Z$ is a random measure on $(U, \mathcal{U})$ with $\mu$ its corresponding signed measure on $\mathrm{U} \times \mathrm{U}$. It is al so clear that $K$ is an inner product preserving isomorphism between $H(Z)(=H(X))$ and $\Lambda_{2}(\mu)$ such that for all $g \in \Lambda_{2}(\mu)$, $K g=\int_{U} g d Z$. Then for all $t \in T$,

$$
X_{t}=\sum_{n=1}^{\infty} a_{n}(t) \xi_{n}=K\left(\sum_{n=1}^{\infty} a_{n}(t) f_{n}\right),
$$

where $a_{n}(t)=E\left[X_{t} \xi_{n}\right], \Sigma_{n=1}^{\infty} a_{n}^{2}(t)=R(t, t)<\infty$, and if we define $f(t, \cdot) \epsilon$ $\Lambda_{2}(\mu)$ by $f(t, u)=\sum_{n=1}^{\infty} a_{n}(t) f_{n}(u)$, we have $X_{t}=K f(t, \cdot)=\int_{U} f(t, u) d Z(u)$ which completes the proof.

Remark 2. In [11] a second order process $X_{t^{\prime}} t \in R^{1}=(-\infty,+\infty)$, is called oscillatory if it has a representation $X_{t}=\int_{-\infty}^{\infty} e^{i t u} a_{t}(u) d Z(u)$ for all $t$ in $R^{1}$, where $Z$ is an orthogonal random measure on $B\left(R^{1}\right)$ with corresponding measure $\nu$ and $a_{t}(\cdot) \in L_{2}(\nu)$ for all $t$ in $T$ (this is a generalization of a more restrictive but physically more significant concept originally introduced in [14]). If $X_{t}, t \in R^{1}$, is oscillatory then $H(X)$ is separable since $L_{2}\left(R^{1}, \mathcal{B}\left(R^{1}\right), \nu\right)$ is separable. Conversely, if $H(X)$ is separable, it follows by Theorem 2(i) that for any finite measure $\nu$ on $\mathscr{P}_{(}\left(R^{1}\right)$ we have $X_{t}=\int_{-\infty}^{\infty} f(t, u) d Z(u)$. for all $t$ in $T$, where $Z$ is an orthogonal random measure on $\mathcal{P}_{(}\left(R^{1}\right)$ with corresponding measure $\nu$, and $f(t, \cdot) \in L_{2}(\nu)$ for all $t$ in $T$. If we define $a_{t}(u)=e^{-i t u} f(t, u)$, it becomes clear that $X_{t}, t \in R^{1}$, is oscillatory. Thus a second order process is oscillatory if and only if its linear space is separable.

\section{REFERENCES}

1. N. Bourbaki, Éléments de mathématique. Livre VI: Intégration. Chaps. I-IX, Actualités Sci. Indust., nos. 1175, 1343, Hermann, Paris, 1952, 1969. MR 14, 960; 43 \#2183. 
2. K. L. Chung and J. L. Doob, Fields, optionality and measurability, Amer. J. Math. 87 (1965), 397-424. MR $35 \# 4972$.

3. D. L. Cohn, Measurable choice of limit points and the existence of separable and measurable processes, Z. Wahrscheinlichkeitstheorie und Verw. Gebiete 22 (1972), 161-165. MR 46 \# 4574.

4. H. Cramér, $A$ contribution to the theory of stochastic processes, Proc. Second Berkeley Sympos. Math. Statist. and Probability (1950), Univ. of California Press, Berkeley, Calif., 1951, pp. 329-339. MR 13, 475.

5. H. Cramér and M. R. Leadbetter, Stationary and related stochastic processes. Sample function properties and their applications, Wiley, New York, 1967. MR 36 $\# 949$.

6. M. M. Crum, On positive-definite functions, Proc. London Math. Soc (3) 6 (1956), 548-560. MR 18, 722.

7. J. L. Doob, Stochastic processes, Wiley, New York; Chapman and Hall, London, 1953. MR 15, 445.

8. N. Dunford and J. T. Schwartz, Linear operators. I: General theory, Pure and Appl. Math., vol. 7, Interscience, New York, 1958. MR 22 \#302.

9. E. Hewitt and K. Stromberg, Real and abstract analysis. A modern treatment of the theory of functions of a real variable, Springer-Verlag, New York, 1965. MR 32 \# 5826.

10. J. Hoffman-Jorgensen, Existence of measurable modifications of stochastic processes, Z. Wahrscheinlichkeitstheorie und Verw. Gebiete 25 (1973), 205207.

11. V. Mandrekar, A characterization of os cillatory processes and their prediction, Proc. Amer. Math. Soc. 32 (1972), 280-284. MR 46 \# 6430.

12. J. Neveu, Processus aléatoires gaussiens, Séminaire de Mathématiques Supérieures, no. 34 (Été, 1968), Les Presses Univ. Montréal, Montréal, Quebec, 1968. MR $42 \# 6923$.

13. E. Parzen, Time series analysis papers, Holden-Day, San Francisco, Calif., 1967. MR 36 \# 6091 .

14. M. B. Priestley, Evolutionary spectra and non-stationary processes, J. Roy. Statist. Soc. Ser. B 27 (1965), 204-237. MR 33 \#8026.

15. Yu A. Rozanov, Stationary random processes, Holden-Day, San Francisco, Calif., 1967. MR 35 \# 4985.

DEPARTMENT OF STATISTICS, UNIVERSITY OF NORTH CAROLINA, CHAPEL HILL, NORTH CAROLINA 27514 